\title{
Suchtmedizin als Teil oder Ganzes, Querschnittsdisziplin oder eigenes Fach?
}

\begin{abstract}
Zusammenfassung: Bei der Sucht handelt es sich um eine behandlungsbedürftige Krankheit, die wie jede andere Krankheit auch mit allen zur Verfügung stehenden Mitteln behandelt werden muss. Da der erste Ansprechpartner eines Suchtkranken in den meisten Fällen der Hausarzt ist, müssen möglichst viele Ärztinnen und Ärzte entsprechend qualifiziert werden, diese Patienten angemessen behandeln zu können. 1998 wurde von der Bundesärztekammer die Fachkunde „Suchtmedizinische Grundversorgung“ eingeführt, die in einem 50-Stunden-Kurs Kenntnisse in der Prävention, Diagnostik, Therapie und Frührehabilitation von den verschiedenen Suchtkrankheiten vermitteln soll. Suchtmedizin ist daher als ein typisches Querschnittsfach anzusehen, das nur unter Einbeziehung aller an der Behandlung Suchtkranker beteiligten Berufsgruppen und medizinischen Disziplinen zu einem wirklich tragfähigen Behandlungskonzept kommen kann.
\end{abstract}

Schlüsselwörter: Suchtmedizinische Versorgung Substitutionstherapie - Fachkunde „Suchtmedizinische Grundversorgung“" Qualitätssicherung

Medical Treatment Of Substance Abuse - A Cross-Sectional Task Or A Speciality Of Its Own?: Substance abuse is a disease that needs optimal medical treatment like any other disease. Because general practitioners are usually the first of the medical profession to start treatment of addicted patients as many physicians as possible should gain the qualification to perform a treatment according to scientific standards. In 1998 the German Medical Association introduced „primary care of drug abuse“ as a complementary specialization to empower as many physicians as possible to treat addicts of all kinds and to initiate early intervention programmes. In an instructional course of 50 hrs knowledge on prevention, diagnostics, therapy and rehabilitation of substance abuse of different kinds is provided. Medical treatment of substance abuse is a typical cross-sectional task only reasonable in a multidisciplinary approach involving all professions and medical specialities dealing with addiction.

Key words: Substance Abuse - Particular Training „Medical Treatment of Substance Abuse“ - Quality Assurance

Suchttherapie Sonderheft 2001; 2: S3-S5

(c) Georg Thieme Verlag Stuttgart · New York ISSN 1439-9903
Ingo Flenker

Ärztekammer Westfalen-Lippe, Ausschuss Sucht und Drogen der Bundesärztekammer

\section{Einleitung}

Eine der großen Herausforderungen in unserer Gesellschaft ist die Behandlung von suchtkranken Menschen - neben den großen chronischen Volkskrankheiten wie Herz-Kreislauferkrankungen und Diabetes werden Suchterkrankungen in Zukunft einen großen Anteil im Behandlungsspektrum gerade des niedergelassenen Arztes darstellen. Dass es sich bei der Sucht um eine behandlungsbedürftige Krankheit handelt, ist mittlerweile unstrittig und dementsprechend haben sich in der Vergangenheit unterschiedliche Ansätze in der Behandlung Suchtkranker entwickelt. Sowohl die Suchterkrankung selber als auch die Gründe für ihre Entstehung sind so vielschichtig und haben so unterschiedliche Ausprägungen, dass nur mit einem breit gefächerten Präventions- und Therapieangebot eine wirkungsvolle Behandlung gewährleistet werden kann.

In den letzten Jahren hat die Behandlung Suchtkranker durch die Ergänzung von medikamentösen und schadensminimierenden Therapieansätzen eine ganz entscheidende Weiterentwicklung erfahren. Ein eigenständiges Fach „Suchtmedizin“ mit verbindlichen Standards und normierten Qualifikationen ist dabei bisher jedoch noch nicht entstanden.

Dabei sind die entsprechenden medizinischen Kenntnisse, Qualifikationen und Kompetenzen auf allen Versorgungsebenen und in allen Versorgungsbereichen von ganz entscheidender Bedeutung. Dies gilt insbesondere für die hausärztlichen Kolleginnen und Kollegen, denen bei der Prävention, Erkennung und Erstbehandlung von Suchterkrankungen eine ganz zentrale Rolle zufällt. Häufig sind sie der erste Ansprechpartner, bei denen der Suchtkranke Rat und Hilfe sucht. Man geht davon aus, dass $75 \%$ aller Personen mit Alkoholproblemen wenigstens einmal im Jahr einen niedergelassenen Arzt und etwa $25 \%$ ein Krankenhaus aufsuchen. Wollen wir also ein Fach „Suchtmedizin“ stärker etablieren, muss es so angelegt sein, dass zunächst durch eine breite Basisqualifikation möglichst viele Ärztinnen und Ärzte in die Lage versetzt werden, Suchtkranke mit allen Abhängigkeitsformen behandeln und eine Frühintervention einleiten zu können. Darauf aufbauend könnte dann mit einem modularen Konzept eine Weiterqualifizierung stattfinden.

Doch würde es der Komplexität dieses Krankheitsbildes nicht entsprechen, wenn man die Behandlung Suchtkranker nur auf den medizinischen Bereich reduzieren würde. Zwar wird die medizinische Behandlung etwa eines Schwerstabhängigen zunächst einmal das Überleben sichern können. Aber erst mit 
der notwendigen psychosozialen Betreuung wird der Patient auch so weit sozial stabilisiert werden können, dass ein Leben außerhalb des Drogenmilieus und eine soziale Wiedereingliederung möglich wird. In den meisten Fällen ist eine Suchterkrankung mit sozialen Problemen verbunden, wie z.B. Wohnungslosigkeit, Arbeitslosigkeit, Straffälligkeit und sozialer Isolation. Die Stärkung des sozialen Umfeldes muss ebenso Bestandteil einer Suchtbehandlung sein wie die gesundheitliche Stabilisierung.

\section{Kooperation und Vernetzung}

Eine wirkungsvolle suchtmedizinische Versorgung ist daher stets auf die Kooperation und Vernetzung mit anderen Berufsgruppen und Einrichtungen angewiesen. Eine multiprofessionelle und disziplinenübergreifende Zusammenarbeit ist in der Versorgung Suchtkranker unerlässlich, um die notwendige umfassende Behandlung und oft schwierige und langfristige Betreuung dieser Patientengruppe zu gewährleisten. Es bedarf der Einrichtung örtlicher Netzwerke, die eine auf breiter Basis stehende fachübergreifende Arbeit möglich machen. Nur so wird die Versorgungsqualität und -effizienz gesteigert werden können. Kooperationstage als Forum für berufsübergreifende Fortbildung, wie Anfang des Jahres erstmalig in Nordrhein-Westfalen durchgeführt, unterstützen dieses Anliegen.

Ohne Zweifel ist die Suchtmedizin als ein typisches Querschnittsfach anzusehen, das nur unter Einbeziehung aller an der Behandlung Suchtkranker beteiligten Berufsgruppen und medizinischen Disziplinen zu einem wirklich tragfähigen Behandlungskonzept kommen kann. Bestes Beispiel für diesen übergreifenden Gedanken ist die Substitutionstherapie, deren Erfolg auf die Fähigkeit und Bereitschaft aller Beteiligten zur professionenübergreifenden Zusammenarbeit zurückzuführen ist. Durch die Substitutionstherapie wurden auch und gerade die niedergelassenen Ärztinnen und Ärzte stärker als bisher in die Versorgung Suchtkranker einbezogen und der Handlungsspielraum der Suchtmedizin über eine stationäre Entgiftung und psychotherapeutische Behandlung hinaus erweitert. Interessanterweise sind es im ambulanten Bereich vorwiegend die niedergelassenen Allgemeinmediziner und weniger die Psychiater, die hier eine zentrale Rolle bei der Substitutionsbehandlung übernommen haben.

In der Zusammenführung der verschiedenen ärztlichen Disziplinen im Rahmen der Suchtbehandlung sehe ich eine wichtige Aufgabe, um einem Fach „Suchtmedizin“ gerecht werden zu können. Die Ärztekammer Westfalen-Lippe hat schon früh begonnen, hier die entsprechenden strukturellen Rahmenbedingungen zu schaffen. Bereits im Jahr 1991 wurde die Beratungskommission „Sucht und Drogen“ bei der Ärztekammer Westfalen-Lippe eingerichtet, die gemeinsam mit Fachleuten der unterschiedlichsten ärztlichen Disziplinen aus dem klinischen und niedergelassenen Bereich sowie Vertretern aus der Drogenhilfe die Zusammenarbeit aller an der Substitution Beteiligten koordiniert. Daneben leistet sie qualifizierte Einzelfallberatung in rechtlicher wie in fachlicher Hinsicht. Die Beratungskommission trägt damit maßgeblich dazu bei, den Kontakt zwischen Ärzten, Drogenhilfe und den anderen Berufen, die an der Behandlung Suchtkranker beteiligt sind, zu verbessern.
Doch nicht nur die Vernetzung innerhalb der Suchtmedizin muss laufend verbessert werden, sondern auch und gerade die Qualität der Behandlung. Leider wird aus meiner Sicht in der medizinischen Ausbildung noch viel zu wenig Augenmerk auf Suchterkrankungen gelegt, so dass sich viele Ärztinnen und Ärzte in der Behandlung dieser Patienten häufig überfordert fühlen. Um so wichtiger ist es, die Ärztinnen und Ärzte gerade in der Grundversorgung gezielt auf die Behandlung dieses Personenkreises vorzubereiten, insbesondere, was die Früherkennung und strukturierte Kurzintervention betrifft. Nur wenn wir eine breite Basis an niedergelassenen Ärztinnen und Ärzte haben, die auf die Behandlung von Suchterkrankungen vorbereitet und entsprechend qualifiziert sind, werden wir den suchtkranken Menschen ein optimales Versorgungsangebot bieten können. Es macht aus meiner Sicht wenig Sinn, die Behandlung Suchtkranker einigen wenigen Spezialisten vorzubehalten, weil die meisten der Suchtkranken den Weg bis zu diesen Spezialisten häufig gar nicht finden.

\section{Fachkunde Suchtmedizinische Grundversorgung}

Um diesen besonderen Anforderungen der Qualifikation der Ärztinnen und Ärzte bei der Suchtbehandlung Rechnung zu tragen, wurde von der Bundesärztekammer im September 1998 eine Fachkunde „Suchtmedizinische Grundversorgung“ eingeführt, die mittlerweile von allen Ärztekammern eingeführt wurde bzw. deren Inhalte durch entsprechende Fortbildungsveranstaltungen angeboten werden. Damit hat man sich auf Bundesebene für ein einstufiges Verfahren im Bereich der suchtmedizinischen Weiterbildung entschieden und auf die Einführung einer Zusatzbezeichnung „Suchtmedizin“ zunächst verzichtet. $\mathrm{Zu}$ konträr sind dabei auch die Meinungen, ob diese Zusatzbezeichnung als Schwerpunktbildung ausschließlich im Gebiet Psychiatrie oder auch in weiteren Gebieten, wie z.B. Allgemeinmedizin und Innere Medizin, erreichbar sein soll. Um den interdisziplinären Charakter der Suchtmedizin zu unterstreichen, haben wir uns in WestfalenLippe allerdings dazu entschlossen, den Erwerb der Fachkunde „Suchtmedizinische Grundversorgung“ allen Fachgebieten mit Patientenbezug zu öffnen. Denn der Chirurg oder Gynäkologe kann mit Suchterkrankungen ebenso konfrontiert werden wie der Dermatologe oder Augenarzt.

Voraussetzung für die Fachkunde ist die Teilnahme an einem 50-Stunden-Kurs, der Kenntnisse in der Prävention, Diagnostik, Therapie und Frührehabilitation von den verschiedenen Suchterkrankungen vermitteln soll. Als integratives Konzept deckt das Curriculum sowohl den Bereich der legalen wie auch der illegalen Drogen ab, denn die Fachkunde sollte nicht ausschließlich eine Qualifikation zur Methadonsubstitution darstellen, sondern vielmehr alle Abhängigkeits- und Missbrauchsformen umfassen. Die Lerninhalte sind modular aufgebaut und bieten mit ihren fünf Bausteinen die Möglichkeit der individuellen Schwerpunktsetzung.

Einstieg in das Curriculum bietet ein 10-stündiges Grundlagen-Seminar, in dem die existierenden Versorgungssysteme ebenso behandelt werden wie die Entstehung von Süchten und ihre soziokulturelle Bedeutung. Die weiteren drei Bausteine beschäftigen sich mit ausgewählten Suchtstoffen wie Alkohol, Tabak, Medikamente und illegale Drogen, wobei jeweils die Aspekte „Grundlagen“, „Erkennen“, „Behandlung“ 
und „Vermitteln“ im Zentrum stehen. Zur Schwerpunktsetzung kann aus diesen drei Bausteinen ein Wahlthema gewählt werden, mit dem die Arbeit für spezielle Tätigkeitsfelder oder Themen vertieft werden kann.

Besonderer Wert wurde bei dem Curriculum auf die Vermittlung von Gesprächsstrategien gelegt, da es nicht immer einfach sein wird, den Patienten etwa auf eine Alkoholerkrankung anzusprechen. In Kleingruppen unter Einbeziehung von Rollenspiel und Videotraining wird der Umgang mit Patienten praxisnah geübt. Ergänzend dazu wird auch die motivierende Gesprächsführung mit Angehörigen trainiert. Bei der Konzeption des Curriculums hat man sich davon leiten lassen, dass die eigene Einstellung und das eigene Verhalten in einer Art Vorbildfunktion in besonderer Weise die jeweiligen Interventionen beeinflussen können. Dementsprechend werden die Kurse aus einer Mischung von Informationsvermittlung, Selbstwahrnehmung und Reflexion persönlicher Einstellungen, problemorientiertem Lernen und individuellem Kompetenztraining angeboten. Mit diesem Kursangebot leistet die Ärzteschaft einen ganz wesentlichen Beitrag zu einer qualitativ hoch stehenden medizinischen Versorgung Suchtkranker.

\section{Qualitätssicherung in der ambulanten Substitutionstherapie}

In idealer Weise verknüpft wurden die Qualitätssicherungsaspekte sowohl für die Behandlung Suchtkranker als auch für die Förderung der Zusammenarbeit der verschiedenen Berufsgruppen in einem Projekt der Ärztekammer WestfalenLippe, das die Wirksamkeit der Substitutionsbehandlung überprüft und entsprechende Qualitätssicherungsinstrumente entwickelt hat. Das Projekt „Qualitätssicherung in der ambulanten Substitutionstherapie“" wurde im Jahr 2000 gestartet und lief bis zum Sommer dieses Jahres, wobei in Teams von Ärzten, Arzthelferinnen sowie Mitarbeitern von Methadon-Ambulanzen und Drogenhilfe-Einrichtungen Leitlinien und Checklisten erarbeitet wurden, mit denen die praktische Arbeit vor Ort sinnvoll unterstützt werden kann. Die Ergebnisse dieses Projektes mit einem praxisorientierten Handbuch wurden am 25.10.2001 in Münster vorgestellt.

Neben Checklisten zu den Themen Praxisorganisation, Teamentwicklung und regionale Kooperationsnetzwerke sowie Muster von Behandlungsverträgen wird das Handbuch auch Checklisten für die Kernprozesse der Substitutionstherapie und therapeutische Leitlinien enthalten. Außerdem werden Vorschläge für eine Basisdokumentation gemacht und Instrumentarien zur Behandlungsplanung und Fortschrittskontrolle angeboten. Darüber hinaus konnten mit Hilfe dieses Modellprojektes wertvolle Hinweise über die Versorgungsrealität in der ambulanten Substitutionstherapie gesammelt werden. Demnach erfolgt wie bereits angedeutet die schwerpunktmäßige Versorgung von opiatabhängigen Patienten durch niedergelassene Allgemeinmediziner und nur in Einzelfällen durch Psychiater.

In der Zusammenarbeit der verschiedenen Berufsgruppen wurde in diesem Projekt ein ganz wesentlicher Grundsatz der Qualitätssicherung befolgt: nämlich die Betroffenen zu Beteiligten zu machen. Denn die Qualitätsverbesserung muss dort ansetzen, wo der tägliche Kontakt mit den Substitutions- patienten stattfindet: in den Praxen der substituierenden Ärztinnen und Ärzte, in den Methadonambulanzen und in den Beratungsstellen. Die dort Tätigen sind besonders geeignet, den Entwicklungsprozess zu einer ständigen Qualitätsverbesserung der Behandlung zu fördern.

\section{Schlussfolgerung und Ausblick}

Wir haben in Deutschland ein breit gefächertes und flächendeckendes Netz an Behandlungs- und Beratungsstellen sowie Instituts- und Fachambulanzen aufgebaut, um die ganze Bandbreite der vielschichtigen Behandlung Suchtkranker abzudecken. Gleichwohl müssen wir bestrebt sein, dieses Netz kontinuierlich weiter auszubauen, insbesondere im niedrigschwelligen Angebotsbereich. Nur wenn es dem Suchtkranken möglich ist, schnell und ohne bürokratische Hürden die entsprechende Hilfe erhalten zu können, wird es langfristig möglich sein, hier ein angemessenes Versorgungsangebot zu schaffen und die Zahl der Drogentoten zu senken.

Tödlich für die Etablierung eines Faches „Suchtmedizin“ wäre eine weitere bürokratische Überreglementierung in diesem Bereich, die immer mehr Ärztinnen und Ärzte von der Suchtbehandlung abschreckt und nicht zu einer stärkeren Akzeptanz dieses Faches beiträgt. Es wird Aufgabe der Politik sein, den finanziellen und strukturellen Rahmen für eine zukunftsfähige Drogenpolitik, ruhend auf den Säulen Prävention, Therapie und Repression, zu schaffen. Zwar hat die rot-grüne Koalition seit ihrer Regierungsübernahme eine sehr zu begrüßende Trendwende in der Drogenpolitik eingeleitet mit dem Ansatz „Hilfe statt Strafe“. Doch darf es nicht nur bei Lippenbekenntnissen der Politiker bleiben, sondern es muss insbesondere die Finanzierung sowohl der ärztlichen Behandlung als auch der begleitenden psychosozialen Betreuung im Rahmen der gesetzlichen Krankenversicherung endlich sichergestellt werden.

Es muss in der Zukunft ein Hilfesystem geschaffen werden, das alle an der Betreuung von Drogenkranken Beteiligten zusammenführt. Ein solches System wird nur erfolgreich sein, wenn es durch verschiedene Berufsgruppen getragen wird. Dies entspricht der Komplexität des Krankheitsbildes „Sucht“. Weder ärztliches Handeln noch psychosoziale Betreuung allein können aus sich heraus eine Lösung für das komplexe Problem der Drogensucht schaffen. Sollte es überhaupt zu lösen sein, geht es nur in gemeinsamer Zusammenarbeit von Drogenhilfe und Ärzteschaft.

\section{Dr. med. Ingo Flenker}

Ärztekammer Westfalen-Lippe

Ausschuss Sucht und Drogen der Bundesärztekammer

Gartenstraße 210-214

48147 Münster 